\title{
Auditory Extension of User Interfaces
}

\author{
Andrea R. Kennel \\ Institut für Informationssysteme, ETH Zürich \\ CH-8092 Zürich, Switzerland
}

\section{User Interface}

Users communicate with computers via the user interface. Internal objects in computer systems like files, windows, menus and messages are represented in the user interface by different symbols, which are perceived and interpreted by the user.

Today most symbols in the user interface are visual symbols. Blattner [1] and Gaver [2] demonstrate that also auditory symbols can be used. If the screen is overloaded or the user is performing a task where he cannot always watch the screen, auditory symbols are helpful. For visually handicapped users the advantage of auditory symbols is obvious.

\section{Auditory Symbols and Point of Experiments}

The perception of auditory symbols is complementary to the perception of visual symbols. A visual symbol exists in space and over time, an auditory symbols exists in time and over space [2]. Therefore auditory symbols are a useful addition to visual symbols. They can be described by the parameters: characteristic; volume; pitch; duration.

Depending on its duration an auditory symbol represents a internal object continuously or instantaneously. For a continuous representation the status of the internal object is represented during its whole existence by an auditory symbol with a flexible duration. For an instantaneous representation only the beginning of a status is represented by an auditory symbol with a short fix duration.

Which and how many auditory symbols can be distinguished by the user? Which are the minimum and maximum duration of auditory symbols? - For these and other questions ergonomic rules have to be found by user testing. Therefore we are developing a user interface framework, which allows to add and exchange interface symbols in a easy way.

\section{References}

1 Blattner, M., Sumikawa, D. \& Greenberg, R. Earcons and icons: Their structure and common design principles. Human-Computer Interaction 4 (1989), 11.

2 Gaver, W. The SonicFinder: An interface that uses auditory icons. HumanComputer Interaction 4 (1989), 67. 


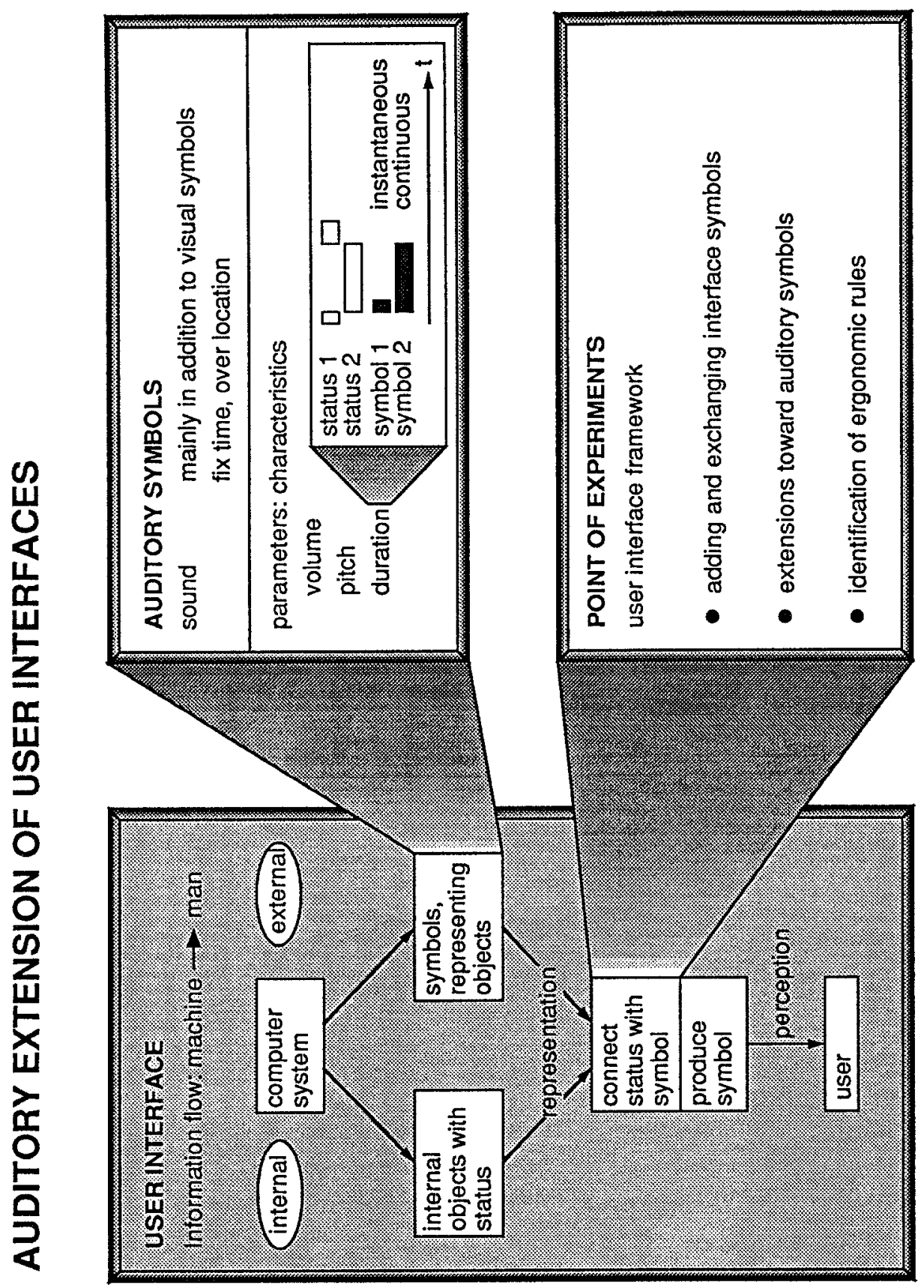

